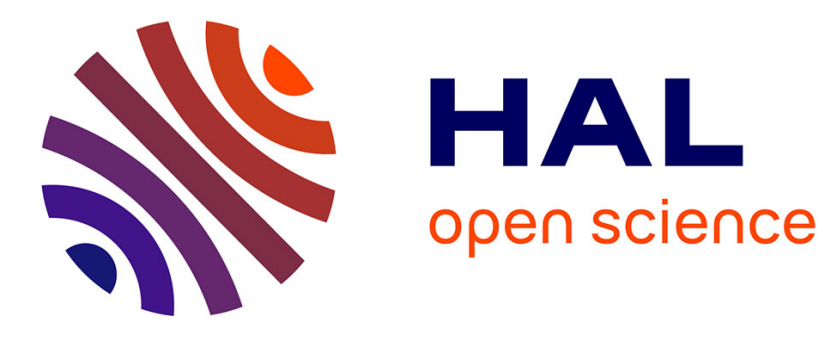

\title{
Nonlinear Regularizing Effect for Conservation Laws
}

\author{
François Golse
}

\section{To cite this version:}

François Golse. Nonlinear Regularizing Effect for Conservation Laws. 12th International Conference on Hyperbolic Problems: Theory, Numerics and Applications, Jun 2008, College Park, Maryland, United States. pp.73-92. hal-00472426

\section{HAL Id: hal-00472426 \\ https://hal-polytechnique.archives-ouvertes.fr/hal-00472426}

Submitted on 12 Apr 2010

HAL is a multi-disciplinary open access archive for the deposit and dissemination of scientific research documents, whether they are published or not. The documents may come from teaching and research institutions in France or abroad, or from public or private research centers.
L'archive ouverte pluridisciplinaire HAL, est destinée au dépôt et à la diffusion de documents scientifiques de niveau recherche, publiés ou non, émanant des établissements d'enseignement et de recherche français ou étrangers, des laboratoires publics ou privés. 


\title{
Nonlinear Regularizing Effect for Conservation Laws
}

\author{
François Golse
}

\begin{abstract}
Compactness of families of solutions - or of approximate solutions - is a feature that distinguishes certain classes of nonlinear hyperbolic equations from the case of linear hyperbolic equations, in space dimension one. This paper shows that some classical compactness results in the context of hyperbolic conservation laws, such as the Lax compactness theorem for the entropy solution semigroup associated with a nonlinear scalar conservation laws with convex flux, or the Tartar-DiPerna compensated compactness method, can be turned into quantitative compactness estimates - in terms of $\epsilon$-entropy, for instance - or even nonlinear regularization estimates. This regularizing effect caused by the nonlinearity is discussed in detail on two examples: a) the case of a scalar conservation law with convex flux, and b) the case of isentropic gas dynamics, in space dimension one.
\end{abstract}

\section{Motivations}

Consider a parabolic PDE of the form

$$
\left\{\begin{aligned}
\partial_{t} u+\partial_{x} f(u) & =\epsilon \partial_{x}^{2} u, \quad x \in \mathbf{R}, t>0 \\
\left.u\right|_{t=0} & =u^{i n}
\end{aligned}\right.
$$

with unknown $u \equiv u(t, x) \in \mathbf{R}$, and $f \in C^{1}(\mathbf{R})$.

For each $\epsilon>0$, the energy equality

$$
\int_{\mathbf{R}} \frac{1}{2} u(t, x)^{2} d x+\epsilon \int_{0}^{t} \int_{\mathbf{R}} \partial_{x} u(s, x)^{2} d x d s=\int_{\mathbf{R}} \frac{1}{2} u^{i n}(x)^{2} d x
$$

implies that $u \in L_{t}^{\infty}\left(L_{x}^{2}\right) \cap L_{t}^{2}\left(\dot{H}_{x}^{1}\right)$ for each $\epsilon>0$ - where $\dot{H}^{1}$ designates the homogeneous Sobolev space. Hence, for each $\epsilon>0$, the solution dynamics

$$
u^{i n} \mapsto u(t, \cdot) \text { maps } L_{x}^{2} \text { into } \dot{H}_{x}^{1} \text { for a.e. } t>0 .
$$

The energy equality above also implies that, for each $\epsilon>0$, the solution map

$$
L_{x}^{2} \ni u^{i n} \mapsto u \in L_{l o c}^{2}(d t d x)
$$

1991 Mathematics Subject Classification. Primary 35L60; Secondary 35B65, 76N15.

Key words and phrases. Hyperbolic systems; $\epsilon$-Entropy, Compensated compactness, Regularizing effect; Scalar conservation law; Isentropic Euler system. 
is compact by the Rellich theorem. In other words, for each bounded $B \subset L_{x}^{2}$ and each $K \subset \mathbf{R}_{+} \times \mathbf{R}$ compact, the set

$$
\left\{\left.u\right|_{K} \mid u^{i n} \in B\right\} \text { is relatively compact in } L^{2}(K ; d t d x) \text {. }
$$

The energy dissipation term

$$
\epsilon \int_{0}^{t} \int_{\mathbf{R}} \partial_{x} u(s, x)^{2} d x d s
$$

is obviously at the origin of the parabolic smoothing mechanism in the viscous conservation law above.

What does remain of this regularizing effect or compactness in the limit as $\epsilon \rightarrow 0^{+}$?

Obviously nothing in the case where $f$ is linear, which leads to a free transport equation of the form

$$
\partial_{t} u+c \partial_{x} u=0 .
$$

The method of characteristics shows that

$$
u(t, x)=u(0, x-c t),
$$

so that $u(t, \cdot)$ cannot be more regular than $u^{i n}$ — in fact, $u(t, \cdot)$ and $u^{i n}$ have exactly the same regularity.

Let us discuss the same question in the nonlinear case, and for entropy solutions of the inviscid equation - indeed, entropy solutions of the inviscid equation are obtained from limits of solutions of the viscous equations in the vanishing viscosity limit. Consider the conservation law for $\epsilon=0$ :

$$
\left\{\begin{aligned}
\partial_{t} u+\partial_{x} f(u) & =0, \quad x \in \mathbf{R}, t>0 \\
\left.u\right|_{t=0} & =u^{i n}
\end{aligned}\right.
$$

with strictly convex flux $f \in C^{1}(\mathbf{R})$ such that $f^{\prime}(z) \rightarrow \pm \infty$ as $z \rightarrow \pm \infty$.

There are two remarkable compactness results for this Cauchy problem, that are similar to the one obtained in the parabolic case:

a) in his 1954 paper L1954 (see p. 190), P. Lax proves that, for each $t>0$, the entropy solution dynamics

$$
u^{i n} \mapsto u(t, \cdot)
$$

is compact from $L_{x}^{1}$ into $L_{l o c}^{1}(d x)$;

b) L. Tartar proved [T] the convergence of the vanishing viscosity method for conservation laws, by using compensated compactness $[\mathbf{M}, \mathbf{T}]$ - the div-curl lemma - together with the entropy bound satisfied by solution of the parabolic approximation.

Both results are based on the fact that

$$
u_{n} \rightarrow u \quad \text { and } \quad F\left(u_{n}\right) \rightarrow F(u)
$$

in the weak-* topology of $L^{\infty}$ and for some appropriate class of nonlinearities $F$ implies that

$$
u_{n} \rightarrow u \quad \text { strongly in } L_{l o c}^{p} \text { for } 1 \leq p<\infty .
$$

Unlike the parabolic case, where compactness in the strong $L^{2}$ topology follows from the $H^{1}$ bound entailed by energy dissipation and the Rellich compactness theorem, this is an example of compactness by nonlinearity: strong convergence results from the commutation of weak convergence with some appropriate nonlinearity. The 
nonlinearity used in the compensated compactness method is the so-called Tartar equation.

In 2002, P. Lax raised the following question: can one transform compactness arguments such as a) above into quantitative compactness estimates? His interest for this question came from the numerical analysis of conservation laws - we shall return to this in the next section.

Since the DiPerna-Tartar compensated compactness method is, so far, the only strategy for obtaining the strong relative compactness of families of solutions and so little seems to be known about the regularity of entropy solutions of systems of conservation laws, it is also fairly natural to ask oneself whether the compensated compactness method can be strengthened into a nonlinear regularization estimate.

A crucial step in turning compensated compactness into a tool for proving nonlinear smoothing is a coercivity estimate satisfied by the Tartar equation - see Lemma 2.3 in section 2. A similar coercivity exists in the case of the isentropic Euler system: see Fact \#1 in section 3 .

\section{1. $\epsilon$-Entropy estimate for scalar conservation laws}

This section reports joint work in collaboration with C. DeLellis [DL-G].

Let $f \in C^{2}(\mathbf{R})$ with $f^{\prime \prime} \geq a>0$, and assume without loss of generality that $f(0)=f^{\prime}(0)=0$. Consider the Cauchy problem

$$
\left\{\begin{aligned}
\partial_{t} u+\partial_{x} f(u) & =0, \quad x \in \mathbf{R}, t>0, \\
\left.u\right|_{t=0} & =u^{i n} .
\end{aligned}\right.
$$

By using different methods, P. Lax L1954, L1957 and O. Oleinik O construct a unique, global weak solution of the Cauchy problem above. Among all weak solutions of this Cauchy problem, this weak solution is the only one satisfying the Lax-Oleinik one-sided estimate

$$
\partial_{x} u \leq \frac{1}{a t}, \quad \text { in the sense of distributions for } t>0 \text { and } x \in \mathbf{R}
$$

and is called the entropy solution of that problem. The entropy solution of the Cauchy problem is related to the initial data $u^{i n}$ by a nonlinear semigroup $(S(t))_{t \geq 0}$.

As recalled in the introduction, P. Lax [L1954, L1957] observed that the entropy semigroup corresponding with the scalar conservation law above satisfies the following compactness property: for each $t>0$, each bounded $B \subset L^{1}(\mathbf{R})$ and each $R>0$, the set

$$
\left\{\left.S(t) f\right|_{(-R, R)} \text { with } f \in B\right\}
$$

is relatively compact in $L^{1}((-R, R))$.

Henceforth, we seek to quantify this compactness result by using the notion of $\epsilon$-entropy, recalled below.

Definition 1.1 (Kolmogorov-Tikhomirov [KT]). For $\epsilon>0$, the $\epsilon$-entropy of $E$ precompact in the metric space $(X, d)$ is :

$$
H_{\epsilon}(E \mid X)=\log _{2} N_{\epsilon}(E)
$$

where $N_{\epsilon}(E)$ is the minimal number of sets in an $\epsilon$-covering of $E$ - i.e. of a covering of $E$ by sets of diameter $\leq 2 \epsilon$ in $X$

Example 1.2. For instance, $H_{\epsilon}\left([0,1]^{n} \mid \mathbf{R}^{n}\right) \simeq n\left|\log _{2} \epsilon\right|$ as $\epsilon \rightarrow 0^{+}$. 
With this definition, we present our quantitative estimate based on the Lax compactness result recalled above.

Theorem 1.3 (DeLellis-Golse, [DL-G]). For each $\epsilon>0$, one has

$$
H_{\epsilon}\left(S(t) \overline{B_{L^{1}(\mathbf{R})}(0, m)} \mid L^{1}([-R, R]) \leq \frac{C_{1}(t)}{\epsilon}+2 \log _{2}\left(\frac{C_{2}(t)}{\epsilon}+C_{3}(t)\right)\right.
$$

where

$$
\begin{array}{r}
C_{1}(t)=\frac{32 R^{2}}{a t}+32 R M(t), \quad C_{3}(t)=3+\frac{2 t M(t) c_{M}(t)}{R+\sqrt{m a t}}, \\
C_{2}(t)=\frac{8 R}{a t}\left(R+\sqrt{m a t}+2 t M(t) c_{M(t)}\right),
\end{array}
$$

and with the notations

$$
M(t)=\sqrt{\frac{4 m}{a t}} \quad \text { and } c_{M}=\sup _{|z| \leq M} f^{\prime \prime}(z) .
$$

The proof of this result uses essentially two ingredients.

To begin with, the entropy semigroup satisfies the following regularizing property: $S(t)$ maps bounded subsets of $L^{1}(\mathbf{R})$ into bounded subsets of $L^{\infty}(\mathbf{R})$ for each $t>0$. More precisely, for each $u^{i n} \in L^{1}(\mathbf{R})$, one has the following estimate:

$$
\left\|S(t) u^{i n}\right\|_{L^{\infty}(\mathbf{R})} \leq \sqrt{\frac{2\left\|u^{i n}\right\|_{L^{1}(\mathbf{R})}}{a t}}, \quad t>0 .
$$

This estimate, obtained by P. Lax in $[\mathbf{L 1 9 7 3}$ is a rather direct consequence of his explicit formula for the entropy solution of a scalar conservation law with convex flux in space dimension one - see also Proposition 1.1 in [DL-G.

The second ingredient in the proof is an estimate of the $\epsilon$-entropy in $L^{1}((0, L))$ of the class

$$
\mathcal{I}_{L, M, V}=\left\{w:[0, L] \rightarrow[0, M] \text { nondecreasing s.t. } w\left(L^{-}\right)-w\left(0^{+}\right) \leq V\right\} .
$$

One finds that

$$
H_{\epsilon}\left(\mathcal{I}_{L, M, V} \mid L^{1}((0, L))\right) \leq 4\left[\frac{L V}{\epsilon}\right]+2 \log _{2}\left[\frac{L M}{\epsilon}+\frac{M}{V}+2\right]
$$

whenever $0<\epsilon \leq \frac{L V}{6}$.

Using these two ingredients together with the Lax-Oleinik one-sided estimate recalled above, we arrive at the bound for the $\epsilon$-entropy presented in the theorem.

We do not know whether our bound for $H_{\epsilon}\left(S(t) \overline{B_{L^{1}(\mathbf{R})}(0, m)} \mid L^{1}([-R, R])\right.$ is sharp. However, the following observation may be relevant.

In his fundamental paper [L1957], P. Lax describes the asymptotic behavior of the entropy solution $S(t) u^{i n}$ in the long time limit $t \rightarrow+\infty$. Specifically, he proves that

$$
S(t) u^{i n}-N_{p, q}(t, \cdot) \rightarrow 0 \text { in } L^{1}(\mathbf{R}) \text { as } t \rightarrow+\infty,
$$

where $N_{p, q}$ is the $\mathrm{N}$-wave defined by the following formula

and where

$$
N_{p, q}(t, x)=\left\{\begin{array}{cc}
x / f^{\prime \prime}(0) t & \text { if }-\sqrt{p t}<x<\sqrt{q t} \\
0 & \text { otherwise, }
\end{array}\right.
$$

$$
p=-2 f^{\prime \prime}(0) \inf _{y} \int_{-\infty}^{y} u^{i n}, \quad q=2 f^{\prime \prime}(0) \sup _{y} \int_{y}^{\infty} u^{i n}
$$




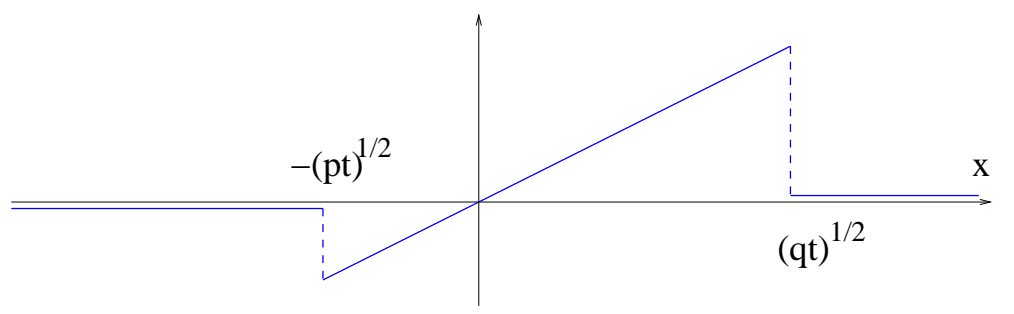

Figure 1. The N-wave $N_{p, q}$

For each $t \geq 0$, set

$$
U_{t}(X)=\sqrt{t} u(t, \sqrt{t} X) \text { where } u(t, \cdot)=S(t) u^{i n} .
$$

Then, the asymptotic behavior of $S(t) u^{i n}$ presented above is equivalent to the limit

$$
U_{t} \rightarrow N_{p, q}(1, \cdot) \text { in } L^{1}(\mathbf{R}) \text { as } t \rightarrow+\infty,
$$

since

$$
\int_{\mathbf{R}} v(x) d x=\int_{\mathbf{R}} \sqrt{t} v(\sqrt{t} X) d X
$$

for each $v \in L^{1}(\mathbf{R})$. The set of all possible limits of $U_{t}$ as $u^{i n}$ runs through $\overline{B_{L^{1}(\mathbf{R})}(0, m)}$ is included in

$$
\mathcal{N}_{m}:=\left\{N_{p, q}(1, \cdot) \mid 0 \leq p, q \leq 2 f^{\prime \prime}(0) m\right\} .
$$

Since $\mathcal{N}_{m}$ is a subset of $L^{1}(\mathbf{R})$ with two degrees of freedom - i.e. depending on the two independent parameters $p, q \in\left[0,2 f^{\prime \prime}(0) m\right]$, one has, as in the example above,

$$
H_{\epsilon}\left(\mathcal{N}_{m} \mid L^{1}(\mathbf{R})\right) \sim 2\left|\log _{2} \epsilon\right|
$$

in the limit as $\epsilon \rightarrow 0^{+}$.

Our bound on the $\epsilon$-entropy does not capture this behavior; yet it shows that

$$
\varlimsup_{t \rightarrow+\infty} H_{\epsilon}\left(S(t) \overline{B_{L^{1}(\mathbf{R})}(0, m)} \mid L^{1}([-R(t), R(t)])\right)=O(1)
$$

as $\epsilon \rightarrow 0^{+}$whenever $R(t)=o(\sqrt{t})$. This asymptotic estimate is consistent with the fact that the dependence of the $N$-wave in $p, q$ can be seen only on intervals of length at least $O(\sqrt{t})$.

In fact, the convergence

$$
U_{t} \rightarrow N_{p, q}(1, \cdot) \text { in } L^{1}(\mathbf{R}) \text { as } t \rightarrow+\infty
$$

is in general not uniform in $u^{i n}$ as $u^{i n}$ runs through $\overline{B_{L^{1}(\mathbf{R})}(0, m)}$. Consider for instance the case of the inviscid Burgers equation

$$
\partial_{t} u+\partial_{x}\left(\frac{1}{2} u^{2}\right)=0
$$

and notice that, for each $\lambda>0$, the rescaled function $(t, x) \mapsto \lambda u\left(\lambda^{2} t, \lambda x\right)$ is a solution of the inviscid Burgers equation whenever $u$ itself is a solution of this equation. For each $u^{i n} \in L^{1}(\mathbf{R})$, set $u_{\lambda}^{i n}(x)=\lambda^{2} u^{i n}(\lambda x)$, so that

$$
\lambda S\left(\lambda^{2} t\right) u_{\lambda}^{i n}=U_{\lambda^{2} t}, \quad t>0,
$$

where the profile $U_{t}$ is defined in terms of the unscaled solution $u(t, \cdot)=S(t) u^{i n}$ as above. One has

$$
\left\|u_{\lambda}^{i n}\right\|_{L^{1}(\mathbf{R})}=\left\|u^{i n}\right\|_{L^{1}(\mathbf{R})}, \quad \lambda>0
$$


and the parameters $p, q$ associated to $u^{i n}$ are left invariant by this scaling.

Because of this scaling, the convergence

$$
U_{\lambda^{2} t} \rightarrow N_{p, q}(1, \cdot) \text { in } L^{1}(\mathbf{R}) \text { as } t \rightarrow+\infty
$$

is not uniform on $\overline{B_{L^{1}(\mathbf{R})}(0, m)}$ for $m \geq\left\|u^{i n}\right\|_{L^{1}(\mathbf{R})}$, since

$$
\sup _{\lambda>0}\left\|U_{\lambda^{2} t}-N_{p, q}(1, \cdot)\right\|_{L^{1}(\mathbf{R})} \geq\left\|U_{1}-N_{p, q}(1, \cdot)\right\|>0,
$$

as can be seen by taking $\lambda=\frac{1}{\sqrt{t}}$. Therefore, one cannot conclude that

$$
\varlimsup_{t \rightarrow+\infty} H_{\epsilon}\left(S(t) \overline{B_{L^{1}(\mathbf{R})}(0, m)} \mid L^{1}(\mathbf{R})\right) \leq H_{\epsilon}\left(\mathcal{N}_{m} \mid L^{1}(\mathbf{R})\right) \sim 2\left|\log _{2} \epsilon\right| .
$$

as $\epsilon \rightarrow 0^{+}$, since the approximation of $U_{\lambda^{2} t}$ by $N_{p, q}(1, \cdot)$ as $t \rightarrow+\infty$ is not uniform on $\overline{B_{L^{1}(\mathbf{R})}(0, m)}$.

Before closing the present section, we recall that P. Lax suggested the idea of using the notion of $\epsilon$-entropy to measure a notion of "resolving power" of a numerical scheme for the conservation law above: see L1978. His conclusion is striking: in his own words, "[...] in the nonlinear case [...] the construction of high resolution methods is easier than in the linear case. It is in this sense that approximating solutions of nonlinear initial value problems is easier than approximating solutions of linear ones."

\section{Regularity by compensated compactness: scalar conservation laws in space dimension 1}

Let $f \in C^{2}(\mathbf{R})$ with $f^{\prime \prime} \geq a>0$, and assume without loss of generality that $f(0)=f^{\prime}(0)=0$; consider then the Cauchy problem

$$
\left\{\begin{aligned}
\partial_{t} u+\partial_{x} f(u) & =0, \quad x \in \mathbf{R}, t>0 \\
\left.u\right|_{t=0} & =u^{i n} .
\end{aligned}\right.
$$

An adaptation of Tartar's compensated compactness method [T] leads to the following regularization estimate.

Theorem 2.1. Assume that $f^{\prime \prime} \geq a>0$, and $f(0)=0$. For each $u^{\text {in }} \in L^{\infty}(\mathbf{R})$ such that $u^{\text {in }}(x)=0$ a.e. in $|x| \geq R$, the entropy solution satisfies the Besov estimate $u \in B_{\infty, l o c}^{1 / 4,4}\left(\mathbf{R}_{+}^{*} \times \mathbf{R}\right)$, i.e.

$$
\int_{0}^{\infty} \int_{\mathbf{R}} \chi(t, x)^{2}|u(t, x)-u(t+s, x+y)|^{4} d x d t=O(|s|+|y|),
$$

for each $\chi \in C_{c}^{1}\left(\mathbf{R}_{+}^{*} \times \mathbf{R}\right)$.

Before sketching the proof of this bound, let us compare the theorem above with previously known results.

First, the Lax-Oleinik one-sided estimate

$$
\partial_{x} u \leq \frac{1}{a t} \text { in the sense of distributions for } t>0 \text { and } x \in \mathbf{R},
$$

implies that $u \in B V_{l o c}\left(\mathbf{R}_{+}^{*} \times \mathbf{R}\right)$. However, this argument is specific to the only case of scalar conservation laws in space dimension 1 , with $f^{\prime \prime} \geq a>0$.

In the case of scalar conservation laws in space dimension higher than one, there is no BV regularization mechanism similar to the case of a convex flux in space dimension one: see Conw on pp. 56-57 for a counter-example. Variants of the 
Lax-Oleinik estimate have been proposed by C. Dafermos [D1985 for degenerate fluxes; analogues in the higher dimensional case are discussed in Chey.

Another method for obtaining a regularization estimate has been proposed by P.-L. Lions, B. Perthame and E. Tadmor LPT-a. They use a kinetic formulation of the scalar conservation law above, together with a velocity averaging regularity estimate. Let us briefly recall their theory: the kinetic formulation of the scalar conservation law

$$
\partial_{t} u+\partial_{x} f(u)=0
$$

is

where

$$
\left(\partial_{t}+f^{\prime}(v) \partial_{x}\right) \chi(u(t, x), v)=\partial_{v} m,
$$

$$
\chi(u, v)= \begin{cases}1 & \text { if } 0<v<u \text { and } 0 \text { if } 0<u<v \\ -1 & \text { if } u<v<0 \text { and } 0 \text { if } v<u<0\end{cases}
$$

and

$$
m \text { is a nonnegative measure on } \mathbf{R}_{+}^{*} \times \mathbf{R} \times \mathbf{R} \text {. }
$$

Velocity averaging regularity bounds are a class of estimates of quantities of the form

$$
\int_{\mathbf{R}} \phi(t, x, v) d v
$$

in Sobolev or Besov spaces with positive regularity exponents, assuming appropriate $L^{p}$-type estimates on both

$$
\phi \equiv \phi(t, x, v) \quad \text { and }\left(\partial_{t}+f^{\prime}(v) \partial_{x}\right) \phi(t, x, v)
$$

whenever $f^{\prime}(v)$ effectively depends on $v$ - this being the case if $f^{\prime \prime}(v) \geq a>0$ for each $v \in \mathbf{R}$ as assumed here. Regularization by velocity averaging was observed for the first time in $\mathbf{A}, \mathbf{G P S}$ and later improved in a series of papers, beginning with GLPS]. More general classes of velocity averaging estimates were later obtained in [DP-L-M], and more recently in [TT. A compendium of velocity averaging regularity bounds can be found in chapter 1 of $\mathbf{B G P}$.

With this method, P.-L. Lions, B. Perthame and E. Tadmor [LPT-a prove that $u \in W_{l o c}^{s, p}\left(\mathbf{R}_{+}^{*} \times \mathbf{R}\right)$ for $s<\frac{1}{3}$ and $1 \leq p<\frac{5}{3}-$ somewhat later, P.-E. Jabin and B. Perthame slightly improved their regularity estimate to all $s<\frac{1}{3}$ and $1 \leq p<\frac{3}{2}$ : see $\mathbf{J P}$.

Although the Lions-Perthame-Tadmor theory based on kinetic formulations and velocity averaging fails to capture the $B V$ regularity predicted by the LaxOleinik theory, it has many advantages over the latter: for one thing, it can be generalized to treat degenerate fluxes, scalar conservation laws in space dimension higher than one, and one very special $2 \times 2$ system in space dimension 1 , namely the isentropic Euler with adiabatic exponent $\gamma=3$ - see below.

Shortly after the Jabin-Perthame paper appeared, C. DeLellis and M. Westdickenberg proved in DL-W that one cannot obtain better regularity than $B_{\infty}^{1 / r, r}$ for $r \geq 3$ or $B_{r}^{1 / 3, r}$ for $1 \leq r<3$ by using only the fact that the entropy production is a bounded Radon measure without using that it is a positive measure.

This remarkable observation explains why all proofs based on kinetic formulations and velocity averaging, such as the Lions-Perthame-Tadmor or the PerthameJabin proof, or on compensated compactness, such as our proof, fail to reach the BV regularity predicted by the Lax-Oleinik one-sided estimate. It also shows that the regularity index $\frac{1}{3}$ in the Lions-Perthame-Tadmor or Jabin-Perthame Sobolev 
estimate is optimal — but not the integrability index $1 \leq p<\frac{5}{2}$. Notice finally that our Besov estimate based on the compensated compactness method, leading to $B_{\infty, l o c}^{1 / 4,4}$ belongs to the optimality class of DeLellis-Westdickenberg (even though the regularity index is $1 / 4$, i.e. less than the optimal value $1 / 3$.)

Unlike the method based on the Lax-Oleinik one-sided estimate, the compensated compactness method allows treating degenerate convex fluxes - as does the method based on velocity averaging (see for instance [TT] on p. 1506).

Assume now that $f \in C^{2}(\mathbf{R})$ satisfies $f(0)=f^{\prime}(0)=0$ and

$$
\left\{\begin{array}{l}
f^{\prime \prime}(v)>0 \text { for each } v \in \mathbf{R} \backslash\left\{v_{1}, \ldots, v_{n}\right\}, \\
f^{\prime \prime}(v) \geq a_{k}\left|v-v_{k}\right|^{2 \beta_{k}} \text { for each } v \text { near } v_{k}, \text { for } k=1, \ldots, n,
\end{array}\right.
$$

for some $v_{1}, \ldots, v_{n} \in \mathbf{R}$ and $a_{1}, \beta_{1}, \ldots, a_{n}, \beta_{n}>0$.

Theorem 2.2. Assume that the flux $f$ satisfies the condition (H) and (without loss of generality) $f(0)=f^{\prime}(0)=0$. For all initial data $u^{\text {in }} \in L^{\infty}(\mathbf{R})$ such that $u^{i n}(x)=0$ a.e. in $|x| \geq R$, the entropy solution $u \in B_{\infty, l o c}^{1 / p, p}\left(\mathbf{R}_{+}^{*} \times \mathbf{R}\right)$, with

$$
p=2 \max _{1 \leq k \leq n} \beta_{k}+4,
$$

i.e.

$$
\int_{0}^{\infty} \int_{\mathbf{R}} \chi(t, x)^{2}|u(t, x)-u(t+s, x+y)|^{p} d x d t=O(|s|+|y|)
$$

for each $\chi \in C_{c}^{1}\left(\mathbf{R}_{+}^{*} \times \mathbf{R}\right)$

However, the interest of compensated compactness as a tool for establishing regularization effects in the context of nonlinear conservation laws is not limited to the scalar case.

Compensated compactness is of course even more promising in the more complex case of systems, for which so little is known about the existence and regularity of solutions in general, and especially $2 \times 2$ hyperbolic systems of conservation laws, since it was used by R. DiPerna in [DP1983b, DP1983a, DP1985] to construct global solutions in the large for a rather general class of such systems. In the case of genuinely nonlinear $2 \times 2$ systems, J. Glimm and P. Lax established some analogue of the Lax-Oleinik estimate, in the case of solutions with small initial oscillations only (see GL, or chapter 12 of D2000.)

In order to understand how Tartar's compensated compactness method can be adapted to establish regularization by nonlinearity in the simplest case of a scalar conservation law with convex flux, we give the proof of Theorem 2.1.

ProOF. We shall only use the fact that the entropy solution $u$ satisfies

$$
\left\{\begin{aligned}
\partial_{t} u+\partial_{x} f(u) & =0, \\
\partial_{t} \frac{1}{2} u^{2}+\partial_{x} g(u) & =-\mu \leq 0,
\end{aligned}\right.
$$

where

$$
g(v):=\int_{0}^{v} w f^{\prime}(w) d w
$$

and $\mu$ is a positive Radon measure such that

$$
\iint_{\mathbf{R}_{+} \times \mathbf{R}} \mu \leq \int_{\mathbf{R}^{2}} \frac{1}{2}\left|u^{i n}\right|^{2} d x<\infty .
$$


Notation: Henceforth, we denote

$$
\tau_{(s, y)} \phi(t, x)=\phi(t-s, x-y), \quad \text { and } J=\left(\begin{array}{cc}
0 & -1 \\
1 & 0
\end{array}\right) .
$$

Step 1: the div-curl argument. Set

$$
B=\left(\begin{array}{c}
u \\
f(u)
\end{array}\right), \quad E=\left(\tau_{(s, y)}-I\right)\left(\begin{array}{c}
\frac{1}{2} u^{2} \\
g(u)
\end{array}\right) .
$$

One has

$$
E, B \in L_{t, x}^{\infty}, \quad \operatorname{div}_{t, x} B=0, \quad \operatorname{div}_{t, x} E=\mu-\tau_{(s, y)} \mu .
$$

In particular, there exists

$$
\pi \in \operatorname{Lip}\left(\mathbf{R}_{+}^{*} \times \mathbf{R}\right), \quad \text { such that } B=J \nabla_{t, x} \pi .
$$

Integrating by parts shows that

$$
\begin{aligned}
\int_{0}^{\infty} \int_{\mathbf{R}} \chi^{2} E \cdot J\left(\tau_{(s, y)} B-B\right) d t d x & =-\int_{0}^{\infty} \int_{\mathbf{R}} \chi^{2} E \cdot \nabla_{t, x}\left(\tau_{(s, y)} \pi-\pi\right) d t d x \\
& =\int_{0}^{\infty} \int_{\mathbf{R}} \nabla_{t, x} \chi^{2} \cdot E\left(\tau_{(s, y)} \pi-\pi\right) d t d x \\
& +\int_{0}^{\infty} \int_{\mathbf{R}} \chi^{2}\left(\tau_{(s, y)} \pi-\pi\right)\left(\mu-\tau_{(s, y)} \mu\right)
\end{aligned}
$$

Therefore, one has the upper bound

$$
\begin{aligned}
\int_{0}^{\infty} \int_{\mathbf{R}} \chi^{2} E & \cdot J\left(\tau_{(s, y)} B-B\right) d t d x \\
\leq & \left(\left\|\nabla_{t, x} \chi^{2}\right\|_{L^{1}}\|E\|_{L^{\infty}}+2\left\|\chi^{2}\right\|_{L^{\infty}} \iint|\mu|\right) \operatorname{Lip}(\pi)(|s|+|y|),
\end{aligned}
$$

which leads to an estimate of the form

$$
\begin{aligned}
\int_{0}^{\infty} \int_{\mathbf{R}} \chi^{2} & \left(\left(\tau_{(s, y)} u-u\right)\left(\tau_{(s, y)} g(u)-g(u)\right)\right. \\
& \left.-\frac{1}{2}\left(\tau_{(s, y)} u^{2}-u^{2}\right)\left(\tau_{(s, y)} f(u)-f(u)\right)\right) d t d x \leq C(|s|+|y|) .
\end{aligned}
$$

Step 2: the coercivity estimate. Next we give a lower bound for the integrand in the left-hand side of the inequality above.

Lemma 2.3. Assume that $f \in C^{2}(\mathbf{R})$ satisfies $f^{\prime \prime}(w) \geq a>0$ for each $w \in \mathbf{R}$. For each $v, w \in \mathbf{R}$, one has

$$
(w-v)(g(w)-g(v))-\frac{1}{2}\left(w^{2}-v^{2}\right)(f(w)-f(v)) \geq \frac{a}{12}|w-v|^{4} .
$$

In [T] — see Remark 30, p. 208 - L. Tartar mentions the inequality

$$
(w-v)(\psi(w)-\psi(v))-(\phi(w)-\phi(v))(f(w)-f(v)) \geq 0, \quad v, w \in \mathbf{R},
$$

whenever $f$ and $\phi$ are convex $C^{1}$ functions defined on $\mathbf{R}$ while $\psi$ is a $C^{1}$ function on $\mathbf{R}$ satisfying

$$
\psi^{\prime}(w)=f^{\prime}(w) \phi^{\prime}(w), \quad w \in \mathbf{R} .
$$

The inequality in the lemma corresponds to the choice

$$
\phi(w)=\frac{1}{2} w^{2}, \quad \psi(w)=g(w) \text { since } g^{\prime}(w)=w f^{\prime}(w), \quad w \in \mathbf{R} .
$$


Proof of Lemma 2.3. Without loss of generality, assume that $v<w$, and write the left-hand side of the inequality above as a double integral:

$$
\begin{aligned}
& (w-v)(g(w)-g(v))-\frac{1}{2}\left(w^{2}-v^{2}\right)(f(w)-f(v))= \\
& \int_{v}^{w} d \xi \int_{v}^{w} \zeta f^{\prime}(\zeta) d \zeta-\int_{v}^{w} \xi d \xi \int_{v}^{w} f^{\prime}(\zeta) d \zeta .
\end{aligned}
$$

Hence

$$
\begin{array}{r}
(w-v)(g(w)-g(v))-\frac{1}{2}\left(w^{2}-v^{2}\right)(f(w)-f(v))=\int_{v}^{w} \int_{v}^{w}(\zeta-\xi) f^{\prime}(\zeta) d \xi d \zeta \\
=\frac{1}{2} \int_{v}^{w} \int_{v}^{w}(\zeta-\xi)\left(f^{\prime}(\zeta)-f^{\prime}(\xi)\right) d \xi d \zeta \geq \frac{a}{2} \int_{v}^{w} \int_{v}^{w}(\zeta-\xi)^{2} d \xi d \zeta
\end{array}
$$

where the second equality follows from symmetrizing the first integral above in $\zeta$ and $\xi$, while the final inequality follows from the mean value theorem and the lower bound $f^{\prime \prime} \geq a>0$.

Step 3: conclusion. Putting together the upper bound for the integral in Step 1 and the lower bound for the integrand of the left hand side obtained in Step 2, we find that

$$
\frac{a}{12} \int_{0}^{\infty} \int_{\mathbf{R}} \chi^{2}\left|\tau_{(s, y)} u-u\right|^{4} d t d x \leq C(|s|+|y|),
$$

which is the announced $B_{\infty, l o c}^{1 / 4,4}$ estimate for the entropy solution $u$.

\section{Regularity by compensated compactness:} the isentropic Euler system in space dimension 1

3.1. The isentropic Euler system. First we recall the Euler system of compressible fluid dynamics for isentropic flows and in space dimension 1.

This is a $2 \times 2$ system, whose unknowns are $\rho \equiv \rho(t, x)$, the fluid density at the position $x$ and at time $t$, and $u \equiv u(t, x)$, the (one-dimensional) velocity field in the fluid at the position $x$ at time $t$.

The isentropic Euler system is

$$
\left\{\begin{array}{l}
\partial_{t} \rho+\partial_{x}(\rho u)=0 \\
\partial_{t}(\rho u)+\partial_{x}\left(\rho u^{2}+\kappa \rho^{\gamma}\right)=0 .
\end{array}\right.
$$

Here, the equation of state for the pressure is

$$
p(\rho)=\kappa \rho^{\gamma}, \quad \rho>0
$$

where $\gamma$ is the adiabatic index and $\kappa>0$ is a constant that can be eliminated by a proper choice of physical units.

In classical gas dynamics, the adiabatic index satisfies $\gamma \geq 1$. It is well known that this condition implies that the isentropic Euler system above is a hyperbolic system with characteristic speeds

$$
\left\{\begin{array}{l}
\lambda_{+} \equiv \lambda_{+}(\rho, u):=u+\theta \rho^{\theta} \\
\lambda_{-} \equiv \lambda_{-}(\rho, u):=u-\theta \rho^{\theta}
\end{array}\right.
$$

Here we have chosen

$$
\kappa=\frac{(\gamma-1)^{2}}{4 \gamma}
$$


so that

henceforth we define

$$
\sqrt{\kappa \gamma}=\frac{\gamma-1}{2}
$$

$$
\theta:=\sqrt{\kappa \gamma}=\frac{\gamma-1}{2}
$$

Whenever the isentropic Euler system has a $C^{1}$ solution $(\rho, u)$, this system can be put in diagonal form as follows

$$
\left\{\begin{array}{l}
\partial_{t} w_{+}(\rho, u)+\lambda_{+}(\rho, u) \partial_{x} w_{+}(\rho, u)=0 \\
\partial_{t} w_{-}(\rho, u)+\lambda_{-}(\rho, u) \partial_{x} w_{-}(\rho, u)=0
\end{array}\right.
$$

where $w_{ \pm}$are the Riemann invariants defined by

$$
\left\{\begin{array}{l}
w_{+}=w_{+}(\rho, u):=u+\rho^{\theta} \\
w_{-}=w_{-}(\rho, u):=u-\rho^{\theta}
\end{array}\right.
$$

R. DiPerna proved in DP1983a that, for each initial data $\left(\rho^{i n}, u^{i n}\right)$ satisfying

$$
\left(\rho^{i n}-\bar{\rho}, u^{i n}\right) \in C_{c}^{2}(\mathbf{R}) \text { and } \rho^{i n}>0,
$$

there exists an entropy (weak) solution $(\rho, u)$ of the isentropic Euler system satisfying the $L^{\infty}$ bound

$$
\begin{aligned}
& 0 \leq \rho \leq \rho^{*}=\sup _{x \in \mathbf{R}}\left(\frac{1}{2}\left(w_{+}\left(\rho^{i n}, u^{i n}\right)-w_{-}\left(\rho^{i n}, u^{i n}\right)\right)^{1 / \theta}\right. \\
& \inf _{x \in \mathbf{R}} w_{-}\left(\rho^{i n}, u^{i n}\right)=: u_{*} \leq u \leq u^{*}:=\sup _{x \in \mathbf{R}} w_{+}\left(\rho^{i n}, u^{i n}\right)
\end{aligned}
$$

DiPerna's argument applied to the case of adiabatic indices of the form

$$
\gamma=1+\frac{2}{2 n+1}, \quad \text { for each } n \in \mathbf{N}^{*} \text {. }
$$

His argument was later improved by G.Q. Chen Chen and, more recently, generalized by P.-L. Lions, B. Perthame, P. Souganidis and E. Tadmor LPT-b, LPS, by using a kinetic formulation of Euler's isentropic system.

PROBLEM: is there a nonlinear regularizing effect for the isentropic Euler system?

3.2. Admissible solutions. An important feature in DiPerna's construction of global entropy solutions of the isentropic Euler system in space dimension 1 is the distinction between weak and strong entropies for that system.

In the present section, it is easier to write the isentropic Euler system in terms of $m=\rho u$, the momentum density:

$$
\left\{\begin{aligned}
\partial_{t} \rho+\partial_{x} m & =0 \\
\partial_{t} m+\partial_{x}\left(\frac{m^{2}}{\rho}+\kappa \rho^{\gamma}\right) & =0 .
\end{aligned}\right.
$$

We recall that an entropy $\phi \equiv \phi(\rho, m)$ of the isentropic Euler system is called a weak entropy if $\left.\phi\right|_{\rho=0}=0$.

An important example of a weak entropy for the isentropic Euler system is the energy

$$
\mathcal{E}(\rho, m):=\frac{1}{2} \frac{m^{2}}{\rho}+\frac{\kappa}{\gamma-1} \rho^{\gamma},
$$


the energy flux being given by the formula

$$
\mathcal{G}(\rho, m)=\frac{m}{\rho}\left(\mathcal{E}(\rho, m)+\kappa \rho^{\gamma}\right) .
$$

DiPerna's solutions are obtained from solutions of the parabolic system

$$
\left\{\begin{array}{r}
\partial_{t} \rho_{\epsilon}+\partial_{x} m_{\epsilon}=\epsilon \partial_{x}^{2} \rho_{\epsilon} \\
\partial_{t} m_{\epsilon}+\partial_{x}\left(\frac{m_{\epsilon}^{2}}{\rho_{\epsilon}}+\kappa \rho_{\epsilon}^{\gamma}\right)=\epsilon \partial_{x}^{2} m_{\epsilon}
\end{array}\right.
$$

- i.e. a compressible Navier-Stokes type system with artificial viscosity $\epsilon>0$ in the limit as $\epsilon \rightarrow 0^{+}$. The solutions $(\rho, m)$ of the isentropic Euler system with $m=\rho u$ obtained in this way satisfy

$$
\partial_{t} \mathcal{E}(\rho, m)+\partial_{x} \mathcal{G}(\rho, m)=-M
$$

where

$$
\epsilon\left(\partial_{x} \rho_{\epsilon}, \partial_{x} m_{\epsilon}\right) \cdot D^{2} \mathcal{E}\left(\rho_{\epsilon}, m_{\epsilon}\right) \cdot\left(\begin{array}{c}
\partial_{x} \rho_{\epsilon} \\
\partial_{x} m_{\epsilon}
\end{array}\right) \rightarrow M \quad \text { in } \mathcal{D}^{\prime}\left(\mathbf{R}_{+}^{*} \times \mathbf{R}\right) \text { as } \epsilon \rightarrow 0^{+} .
$$

One can check that $\mathcal{E}$ is a convex function of $(\rho, m)$ since

$$
D^{2} \mathcal{E}(\rho, m)=\frac{1}{\rho}\left(\begin{array}{cc}
\frac{m^{2}}{\rho^{2}}+\kappa \gamma \rho^{\gamma-2} & \frac{m}{\rho} \\
\frac{m}{\rho} & 1
\end{array}\right) \text { is positive definite, }
$$

so that

$$
M \geq 0 \text { is a Radon measure, }
$$

and the convergence of the dissipation term above holds in the weak topology of Radon measures.

Now, each weak entropy $\phi \equiv \phi(\rho, m)$ has its dissipation dominated by the energy dissipation. Indeed, each weak entropy is of the form

$$
\phi(\rho, m)=A \rho \int_{\mathbf{R}} g\left(\frac{m}{\rho}+\rho^{\theta} z\right)\left(1-z^{2}\right)^{\alpha} d z,
$$

where $g$ is a $C^{2}$ function on $\mathbf{R}$ and

$$
\alpha=\frac{3-\gamma}{2(\gamma-1)}, \quad \frac{1}{A}=\int_{-1}^{1}\left(1-z^{2}\right)^{\alpha} d z .
$$

In particular, for $g(\xi)=\frac{1}{2} \xi^{2}$, one finds

$$
\mathcal{E}(\rho, m)=\frac{A}{2} \rho \int_{\mathbf{R}}\left(\frac{m}{\rho}+\rho^{\theta} z\right)^{2}\left(1-z^{2}\right)^{\alpha} d z .
$$

Then, for each compact $K \subset \mathbf{R}_{+} \times \mathbf{R}$, there exists $C_{\phi, K}>0$ such that

$$
\left|D^{2} \phi(\rho, m)\right| \leq C_{\phi, K} D^{2} \mathcal{E}(\rho, m) \text { for }\left(\rho, \frac{m}{\rho}\right) \in K \text {. }
$$

Hence all DiPerna solutions of Euler's system constructed as above satisfy, for each weak entropy $\phi$, the entropy condition

$$
\partial_{t} \phi(\rho, m)+\partial_{x} \psi(\rho, m)=-\mu[\phi]
$$

where $\mu[\phi]$ is a bounded Radon measure verifying the bound

$$
|\langle\mu[\phi], \chi\rangle| \leq C_{\phi, K}\langle M, \chi\rangle, \quad \chi \in C_{c}^{\infty}\left(\mathbf{R}_{+} \times \mathbf{R}\right)
$$

where we recall that $M$ is the energy dissipation. 
These considerations suggest the following notion of an admissible solution of the isentropic Euler system on an open subset in space-time.

Definition 3.1. Let $\mathcal{O} \subset \mathbf{R}_{+}^{*} \times \mathbf{R}$ open, and $\rho^{*}>\rho_{*}>0$ and $u^{*}>u_{*}$ four constants in $\mathbf{R}$. A weak solution $(\rho, m)$ such that

$$
0<\rho_{*} \leq \rho \leq \rho^{*} \quad \text { and } \quad u_{*} \leq \frac{m}{\rho} \leq u^{*} \text { for each }(t, x) \in \mathcal{O}
$$

is called an admissible solution on $\mathcal{O}$ if and only if, for each entropy $\phi$, weak or not,

$$
\partial_{t} \phi(\rho, m)+\partial_{x} \psi(\rho, m)=-\mu[\phi]
$$

is a Radon measure such that

$$
\|\mu[\phi]\|_{\mathcal{M}_{b}(\mathcal{O})} \leq C\left(\rho_{*}, \rho^{*}, u_{*}, u^{*}\right)\left\|D^{2} \phi\right\|_{L^{\infty}\left(\left[\rho_{*}, \rho^{*}\right] \times\left[u_{*}, u^{*}\right]\right.} \int_{\mathcal{O}} M .
$$

Whether admissible solutions in the large of the isentropic Euler system globally exist on $\mathbf{R}_{+}^{*} \times \mathbf{R}$ seems to be an open question at the time of this writing.

For instance, any DiPerna solution whose viscous approximation $\left(\rho_{\epsilon}, m_{\epsilon}\right)$ satisfies the uniform lower bound

$$
\rho_{\epsilon} \geq \rho_{*}>0 \quad \text { on } \mathcal{O} \text { for each } \epsilon>0
$$

is admissible on $\mathcal{O}$. However, obtaining lower bounds on the density for solutions of the compressible Navier-Stokes system with artificial, diagonal viscosity uniformly as the viscosity $\epsilon \rightarrow 0^{+}$seems to be a nontrivial question.

3.3. Nonlinear regularizing effect for isentropic Euler. The proof of nonlinear regularization by compensated compactness sketched in the previous section can be adapted to the isentropic Euler system, following the work of R. DiPerna DP1983b] who succeded in adapting Tartar's compensated compactness method to treat the case of $2 \times 2$ systems.

Theorem 3.2. Assume that $\gamma \in(1,3)$ and let $\mathcal{O}$ be any open set in $\mathbf{R}_{+}^{*} \times \mathbf{R}$. Any admissible solution $(\rho, m)$ of Euler's isentropic system on $\mathcal{O}$ satisfies

$$
\iint_{\mathcal{O}}|(\rho, u)(t+s, x+y)-(\rho, u)(t, x)|^{2} d x d t \leq \text { Const. }|\ln (|s|+|y|)|^{-2}
$$

whenever $|s|+|y|<\frac{1}{2}$, where $u=\frac{m}{\rho}$.

In the special case $\gamma=3$, the same method gives

Theorem 3.3. Assume that $\gamma=3$ and let $\mathcal{O}$ be any open set in $\mathbf{R}_{+}^{*} \times \mathbf{R}$. Any admissible solution of Euler's isentropic system on $\mathcal{O} \subset \mathbf{R}_{+}^{*} \times \mathbf{R}$ satisfies

$$
(\rho, u) \in B_{\infty, l o c}^{1 / 4,4}(\mathcal{O})
$$

Before sketching the proof of Theorem 3.2, a few remarks are in order.

For $\gamma=3$, by using the kinetic formulation and velocity averaging, P.-L. Lions, B. Perthame and E. Tadmor [LPT-b] obtained a first regularizing effect, later improved by P.-E. Jabin and B. Perthame [JPP], who found that

$$
\rho, \rho u \in W_{l o c}^{s, p}\left(\mathbf{R}_{+} \times \mathbf{R}\right) \text { for all } s<\frac{1}{4}, 1 \leq p \leq \frac{8}{5}
$$

for any entropy solution of the isentropic Euler system — without having to assume any admissibility condition as above. 
The reason why the case $\gamma=3$ is so special in the theory of the isentropic Euler system can be explained as follows.

According to Lions-Perthame-Tadmor [LPT-b], the kinetic formulation of the isentropic Euler system is of the form

$$
\begin{aligned}
\partial_{t} \chi+\partial_{x}[(\theta \xi+(1-\theta) u(t, x)) \chi]=\partial_{\xi}^{2} m, & \text { with } m \geq 0, \\
\text { and } \chi=\left[\left(w_{+}-\xi\right)\left(\xi-w_{-}\right)\right]_{+}^{\alpha}, & \text { for } \alpha=\frac{3-\gamma}{2(\gamma-1)} .
\end{aligned}
$$

Because of the presence of $u(t, x)$ in the advection velocity - which is just bounded, and not smooth - classical velocity averaging lemmas A, GPS, GLPS, DP-L-M, Gér ...- do not apply, except in the case where

$$
\theta=\frac{\gamma-1}{2}=1 \text { or equivalently } \gamma=3 \text {. }
$$

In that case, the kinetic formulation above becomes

$$
\partial_{t} \chi+\xi \partial_{x} \chi=\partial_{\xi}^{2} m, \quad \text { with } m \geq 0,
$$

to which the usual velocity averaging results can be applied without difficulty.

The Lions-Perthame-Tadmor kinetic formulation of the $p$-system for $\gamma \in(1,3]$ was used subsequently by P.-L. Lions, B. Perthame and P. Souganidis, who proved strong compactness for bounded families of entropy solutions for all $\gamma \in(1,3]$, thereby completing the earlier results by R. DiPerna and G.Q. Chen on that problem.

3.4. Proof of nonlinear regularizing effect for isentropic Euler. In this section, we briefly sketch the proof of Theorem 3.2.

The div-curl bilinear inequality. Perhaps the best known example of com-

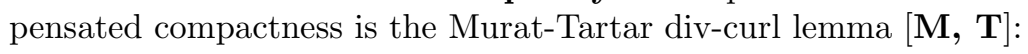

Lemma 3.4 (Div-curl lemma). Let $\Omega$ be an open subset of $\mathbf{R}^{3}$ and $E_{n} \equiv E_{n}(x)$ and $B_{n} \equiv B_{n}(x)$ be two sequences of functions in $L^{2}(\Omega)$ satisfying

$$
\begin{aligned}
& E_{n} \rightarrow E \text { weakly in } L^{2}(\Omega), \\
& B_{n} \rightarrow B \text { weakly in } L^{2}(\Omega),
\end{aligned}
$$

as $n \rightarrow+\infty$, while

$$
\begin{aligned}
& \operatorname{div} E_{n} \text { relatively compact in } H_{l o c}^{-1}(\Omega), \\
& \text { curl } B_{n} \text { relatively compact in } H_{l o c}^{-1}(\Omega)
\end{aligned}
$$

Then

as $n \rightarrow+\infty$.

$$
E_{n} \cdot B_{n} \rightarrow E \cdot B \text { in } \mathcal{D}^{\prime}(\Omega)
$$

A variant of the Murat-Tartar div-curl lemma is the following bilinear estimate, which we state in the two dimensional setting needed in the context of hyperbolic systems of conservation laws.

Let $\mathcal{O}$ be an open subset of $\mathbf{R}_{+}^{*} \times \mathbf{R}$, and let $\chi \in C_{c}^{\infty}(\mathcal{O})$. Let $E \equiv E(t, x) \in \mathbf{R}^{2}$ and $B \equiv B(t, x) \in \mathbf{R}^{2}$ be two vector fields on $\mathcal{O}$ satisfying $E, B \in L^{\infty}\left(\mathcal{O} ; \mathbf{R}^{2}\right)$. Then one has the 


\section{DIV-CURL BILINEAR INEQUALITY}

$$
\begin{aligned}
\left|\iint_{\mathcal{O}} \chi^{2} E \cdot J B d t d x\right| & \leq\|\chi E\|_{L^{p}(\mathcal{O})}\left\|\chi \operatorname{div}_{t, x} B\right\|_{W^{-1, p^{\prime}}(\mathcal{O})} \\
& +\|\chi B\|_{L^{p}(\mathcal{O})}\left\|\chi \operatorname{div}_{t, x} E\right\|_{W^{-1, p^{\prime}}(\mathcal{O})} \\
& +\|\chi E\|_{L^{2}(\mathcal{O})}\left\|\nabla_{t, x} \chi \cdot B\right\|_{H^{-1}(\mathcal{O})} \\
& +\|\chi B\|_{L^{2}(\mathcal{O})}\left\|\nabla_{t, x} \chi \cdot E\right\|_{H^{-1}(\mathcal{O})}
\end{aligned}
$$

where $p \in(1, \infty), p^{\prime}=\frac{p}{p-1}$ is the dual exponent of $p$, and $J$ designates the rotation of an angle $\frac{\pi}{2}$ :

$$
J=\left(\begin{array}{cc}
0 & -1 \\
1 & 0
\end{array}\right) .
$$

How this bilinear inequality is related to the Murat-Tartar div-curl lemma is obvious: if $E_{n} \rightarrow 0$ and $B_{n} \rightarrow 0$ weakly in $L^{2}$, then $E_{n}$ and $B_{n}$ are bounded in $L^{2}$ by the Banach-Steinhaus theorem. Besides, if both $\operatorname{div}_{t, x} E_{n}$ and $\operatorname{div}_{t, x}\left(J B_{n}\right)-$ the two-dimensional analogue of $\operatorname{curl} B_{n}$ - are relatively compact in $H_{l o c}^{-1}$, then

$$
\operatorname{div}_{t, x} E_{n} \text { and } \operatorname{div}_{t, x} J B_{n} \rightarrow 0 \text { in } H_{l o c}^{-1} \text { as } n \rightarrow+\infty .
$$

After replacing $B_{n}$ with $J B_{n}$, the bilinear estimate above implies indeed that

$$
\iint_{\mathcal{O}} \chi^{2} E_{n} \cdot B_{n} d t d x \rightarrow 0
$$

for each $\chi \in C_{c}^{\infty}(\mathcal{O})$ as $n \rightarrow+\infty$, meaning that

$$
E_{n} \cdot B_{n} \rightarrow 0 \text { in } \mathcal{D}^{\prime}(\mathcal{O}) \text { as } n \rightarrow+\infty .
$$

In other words, the bilinear inequality above is a quantitative variant of the Murat-Tartar div-curl compactness lemma.

Returning to the isentropic Euler system, we apply this bilinear inequality with

$$
E=\left(\tau_{(s, y)}-I\right)\left(\begin{array}{l}
\phi_{1}(\rho, u) \\
\psi_{1}(\rho, u)
\end{array}\right) \quad B=\left(\tau_{(s, y)}-I\right)\left(\begin{array}{l}
\phi_{2}(\rho, u) \\
\psi_{2}(\rho, u)
\end{array}\right)
$$

where $\left(\phi_{1}, \psi_{1}\right)$ and $\left(\phi_{2}, \psi_{2}\right)$ are two entropy pairs for the isentropic Euler system, while $(\rho, u)$ is an admissible solution of isentropic Euler on $\mathcal{O}$, and $\operatorname{supp}(\chi)$ is a compact subset of $\mathcal{O}$.

The admissibility condition implies that

$$
\begin{aligned}
\operatorname{div}_{t, x} E & =-\left(\tau_{(s, y)}-I\right) \mu\left[\phi_{1}\right], \\
\operatorname{div}_{t, x} B & =-\left(\tau_{(s, y)}-I\right) \mu\left[\phi_{2}\right],
\end{aligned}
$$

with

$$
\left\|\mu\left[\phi_{j}\right]\right\|_{\mathcal{M}_{b}(\mathcal{O})} \leq C\left\|D^{2} \phi_{j}\right\|_{L^{\infty}\left(\left[\rho_{*}, \rho^{*}\right] \times\left[u_{*}, u^{*}\right]\right)},
$$

where we recall that

$$
\left\{\begin{array}{l}
0<\rho_{*} \leq \rho \leq \rho^{*} \\
u_{*} \leq u=\frac{m}{\rho} \leq u^{*}
\end{array} \quad \text { on } \mathcal{O} .\right.
$$

By Sobolev embedding $W^{r, p}\left(\mathbf{R}^{2}\right) \subset C\left(\mathbf{R}^{2}\right)$ for $r>\frac{2}{p}$; then by duality

$$
\left\|\chi \operatorname{div}_{t, x} E\right\|_{W^{-1, p^{\prime}}} \leq C_{r}\left\|D^{2} \phi_{j}\right\|_{L^{\infty}\left(\left[\rho_{*}, \rho^{*}\right] \times\left[u_{*}, u^{*}\right]\right)}(|s|+|y|)^{1-r}
$$


and likewise for $B$. Applying the bilinear div-curl inequality implies that

$$
\left|\iint \chi^{2} E \cdot J B d t d x\right| \leq C_{r}\left\|D^{2} \phi_{j}\right\|_{L^{\infty}\left(\left[\rho_{*}, \rho^{*}\right] \times\left[u_{*}, u^{*}\right]\right)}(|s|+|y|)^{1-r} .
$$

This first step in the proof of nonlinear regularization for isentropic Euler can be summarized as follows: the div-curl bilinear estimate provides an upper bound on the quantity $E \cdot J B$ arising in Tartar's equation for entropy-entropy flux pairs.

The Tartar equation for Lax entropies. Define

$$
\begin{aligned}
T\left[\phi_{1}, \phi_{2}\right](U, V): & =\left(\phi_{1}(V)-\phi_{1}(U)\right)\left(\psi_{2}(V)-\psi_{2}(U)\right) \\
& -\left(\psi_{1}(V)-\psi_{1}(U)\right)\left(\phi_{2}(V)-\phi_{2}(U)\right), \quad U, V \in \mathbf{R}_{+} \times \mathbf{R}
\end{aligned}
$$

for two entropy pairs $\left(\phi_{1}, \psi_{1}\right)$ and $\left(\phi_{2}, \psi_{2}\right)$, so that

$$
E \cdot J B=T\left[\phi_{1}, \phi_{2}\right]\left(\tau_{(s, y)}(\rho, m),(\rho, m)\right) \text {. }
$$

Therefore, for each $\chi \in C_{c}^{1}(\mathcal{O})$, step 1 leads to an upper bound for

$$
\begin{aligned}
\iint_{\mathcal{O}} \chi^{2} T\left[\phi_{1}, \phi_{2}\right]\left(\tau_{(s, y)}(\rho, u)\right. & ,(\rho, u)) d t d s=\iint_{\mathcal{O}} \chi^{2} E \cdot J B d t d s \\
& \leq C_{r}\left\|D^{2} \phi_{j}\right\|_{L^{\infty}\left(\left[\rho_{*}, \rho^{*}\right] \times\left[u_{*}, u^{*}\right]\right)}(|s|+|y|)^{1-r} .
\end{aligned}
$$

As in the case of a scalar conservation law, we need a lower bound of that same quantity.

We shall analyze the quantity $T\left[\phi_{1}, \phi_{2}\right]$ for a particular class of entropies, the Lax entropies - introduced by Lax in [L1971. These entropies are best expressed in Riemann invariant coordinates $w=\left(w_{+}, w_{-}\right)$:

$$
\begin{aligned}
& \phi_{ \pm}(w, k)=e^{k w_{ \pm}}\left(A_{0}^{ \pm}(w)+\frac{A_{1}^{ \pm}(w)}{k}+\ldots\right), \text { with entropy flux } \\
& \psi_{ \pm}(w, k)=e^{k w_{ \pm}}\left(B_{0}^{ \pm}(w)+\frac{B_{1}^{ \pm}(w)}{k}+\ldots\right), \quad k \rightarrow \pm \infty
\end{aligned}
$$

P. Lax observed that these entropies always exist for strictly hyperbolic systems L1971; this is one of the reasons why we need a uniform lower bound on the fluid density, of the form $\rho \geq \rho_{*}>0$ on $\mathcal{O}$, since the characteristic speeds of the isentropic Euler system satisfy

$$
\lambda_{+}(\rho, u)-\lambda_{-}(\rho, u)=2 \theta \rho^{\theta} .
$$

Let us consider the leading order term in Tartar's equation: as $k \rightarrow+\infty$. With the notation

one has

$$
U=\left(\rho_{1}, m_{1}\right), \quad V=\left(\rho_{2}, m_{2}\right) \in \mathbf{R}_{+}^{*} \times \mathbf{R}
$$

$$
\begin{aligned}
T\left[\phi_{+}(\cdot,+k), \phi_{+}(\cdot,-k)\right](U, V) & =2 A_{0}^{+}(w(U)) A_{0}^{+}(w(V)) \\
& \times\left(\lambda_{+}(U)-\lambda_{+}(V)\right) \sinh \left(k\left(w_{+}(U)-w_{+}(V)\right)\right)+\ldots \\
T\left[\phi_{-}(\cdot,+k), \phi_{-}(\cdot,-k)\right](U, V) & =2 A_{0}^{-}(w(U)) A_{0}^{-}(w(V)) \\
& \times\left(\lambda_{-}(U)-\lambda_{-}(V)\right) \sinh \left(k\left(w_{-}(U)-w_{-}(V)\right)\right)+\ldots
\end{aligned}
$$

At this point, we use two important, special features of Euler's isentropic system. 
FACT \#1.

We recall the notation $\theta=\frac{\gamma-1}{2}$. Then

$$
\left(\begin{array}{l}
\lambda_{+} \\
\lambda_{-}
\end{array}\right)=\mathcal{A}\left(\begin{array}{l}
w_{+} \\
w_{-}
\end{array}\right) \text {with } \mathcal{A}=\frac{1}{2}\left(\begin{array}{cc}
1+\theta & 1-\theta \\
1-\theta & 1+\theta
\end{array}\right)
$$

and for $\gamma \in(1,3)$ one has $\theta \in(0,1)$, leading to the coercivity estimate

$$
\left(\begin{array}{l}
\sinh (a) \\
\sinh (b)
\end{array}\right) \cdot \mathcal{A}\left(\begin{array}{l}
a \\
b
\end{array}\right) \geq \theta(a \sinh (a)+b \sinh (b))
$$

for each $a, b \in \mathbf{R}$. Thus

$$
\begin{aligned}
&\left(\lambda_{+}(U)-\lambda_{+}(V)\right) \sinh \left(k\left(w_{+}(U)-w_{+}(V)\right)\right) \\
&+\left(\lambda_{-}(U)-\lambda_{-}(V)\right) \sinh \left(k\left(w_{-}(U)-w_{-}(V)\right)\right) \\
& \geq \theta\left(\left(w_{+}(U)-w_{+}(V)\right)\right. \sinh \left(k\left(w_{+}(U)-w_{+}(V)\right)\right) \\
&\left.+\left(w_{-}(U)-w_{-}(V)\right) \sinh \left(k\left(w_{-}(U)-w_{-}(V)\right)\right)\right) .
\end{aligned}
$$

This coercivity estimate suggests seeking a lower bound for linear combinations of the quantities $T\left[\phi_{+}(\cdot,+k), \phi_{+}(\cdot,-k)\right]$ and $T\left[\phi_{-}(\cdot,+k), \phi_{-}(\cdot,-k)\right]$ of the form

$$
c_{+}^{2} T\left[\phi_{+}(\cdot,+k), \phi_{+}(\cdot,-k)\right](U, V)+c_{-}^{2} T\left[\phi_{-}(\cdot,+k), \phi_{-}(\cdot,-k)\right](U, V) .
$$

Since the div-curl bilinear estimate presented in the previous section bears on integrals of the terms,

$$
\begin{aligned}
& \chi^{2} T\left[\phi_{+}(\cdot,+k), \phi_{+}(\cdot,-k)\right]\left((\rho, m), \tau_{s, y}(\rho, m)\right) \simeq 2 \chi^{2} A_{0}^{+}(w((\rho, m))) A_{0}^{+}\left(w\left(\tau_{s, y}(\rho, m)\right)\right) \\
& \times\left(\lambda_{+}((\rho, m))-\lambda_{+}\left(\tau_{s, y}(\rho, m)\right)\right) \sinh \left(k\left(w_{+}((\rho, m))-w_{+}\left(\tau_{s, y}(\rho, m)\right)\right)\right) \\
& \chi^{2} T\left[\phi_{-}(\cdot,+k), \phi_{-}(\cdot,-k)\right]\left((\rho, m), \tau_{s, y}(\rho, m)\right) \simeq 2 \chi^{2} A_{0}^{-}(w((\rho, m))) A_{0}^{-}\left(w\left(\tau_{s, y}(\rho, m)\right)\right) \\
& \times\left(\lambda_{-}((\rho, m))-\lambda_{-}\left(\tau_{s, y}(\rho, m)\right)\right) \sinh \left(k\left(w_{-}((\rho, m))-w_{-}\left(\tau_{s, y}(\rho, m)\right)\right)\right)
\end{aligned}
$$

to leading order in $k$ as $|k| \rightarrow \infty$, while the coercivity estimate above bears on

$$
\begin{aligned}
& \left(\lambda_{+}((\rho, m))-\lambda_{+}\left(\tau_{s, y}(\rho, m)\right)\right) \sinh \left(k\left(w_{+}((\rho, m))-w_{+}\left(\tau_{s, y}(\rho, m)\right)\right)\right) \\
+ & \left(\lambda_{-}((\rho, m))-\lambda_{-}\left(\tau_{s, y}(\rho, m)\right)\right) \sinh \left(k\left(w_{-}((\rho, m))-w_{-}\left(\tau_{s, y}(\rho, m)\right)\right)\right),
\end{aligned}
$$

we can hope to use the div-curl bilinear estimate together with this coercivity estimate, provided that the leading order terms in Lax entropies are proportional and positive:

$$
c_{+} A_{0}^{+}(w)=c_{-} A_{0}^{-}(w)>0 .
$$

FACT \#2:

Fortunately, Euler's isentropic system satisfies the relation

$$
\partial_{w_{+}}\left(\frac{\partial_{w-} \lambda_{+}}{\lambda_{+}-\lambda_{-}}\right)=\partial_{w_{-}}\left(\frac{\partial_{w_{+}} \lambda_{-}}{\lambda_{-}-\lambda_{+}}\right) .
$$

Hence there exists a function $\Lambda \equiv \Lambda\left(w_{+}, w_{-}\right)$such that

$$
\nabla \Lambda=\left(\begin{array}{c}
\partial_{w_{+}} \Lambda \\
\partial_{w_{-}} \Lambda
\end{array}\right)=\left(\begin{array}{c}
\frac{\partial_{+} \lambda_{-}}{\lambda_{-}-\lambda_{+}} \\
\frac{\partial_{-} \lambda_{+}}{\lambda_{+}-\lambda_{-}}
\end{array}\right)
$$


so that one can take

$$
A_{0}^{+}\left(w_{+}, w_{-}\right)=A_{0}^{-}\left(w_{+}, w_{-}\right)=e^{\Lambda\left(w_{+}, w_{-}\right)}>0 ;
$$

see for instance fla. (12.2.11) in D2000.

Here we choose

$$
A_{0}^{+}\left(w_{+}, w_{-}\right)=A_{0}^{-}\left(w_{+}, w_{-}\right)=\left(w_{+}-w_{-}\right)^{\frac{1-\theta}{2 \theta}}>0
$$

since the isentropic Euler system is assumed to be uniformly strictly hyperbolic:

$$
w_{+}-w_{-}=2 \rho^{\theta} \geq 2 \rho_{*}^{\theta}>0, \quad \text { on } \mathcal{O} .
$$

Putting together the upper bound obtained from the div-curl bilinear estimate, and the lower bound coming from the coercivity estimate, and optimizing in $k \rightarrow+\infty$ as $|s|+|y| \rightarrow 0^{+}$, one arrives at the logarithmic modulus of continuity announced in Theorem 3.2. A complete proof can be found in $\mathbf{G o}$.

\section{Conclusions and perspectives}

At variance with the original DiPerna argument (1983) for genuinely nonlinear $2 \times 2$ system, the proof of the nonlinear regularizing effect above is based on the coercivity of the leading order term in the Tartar equation — whereas DiPerna's argument uses the next to leading order term in that same equation. More precisely, the proof of nonlinear regularization presented above is based on the coercivity inequality

$$
\left(\begin{array}{l}
\sinh (a) \\
\sinh (b)
\end{array}\right) \cdot \mathcal{A}\left(\begin{array}{l}
a \\
b
\end{array}\right) \geq \theta(a \sinh (a)+b \sinh (b)),
$$

where

$$
\mathcal{A}:=\frac{1}{2}\left(\begin{array}{cc}
1+\theta & 1-\theta \\
1-\theta & 1+\theta
\end{array}\right)=\frac{\partial\left(\lambda_{+}, \lambda_{-}\right)}{\partial\left(w_{+}, w_{-}\right)} .
$$

This condition is stronger than assuming that $\mathcal{A}$ is positive definite - which corresponds to keeping only the leading order terms in the inequality above as $|a|+|b| \rightarrow 0$. In DiPerna's paper [DP1983b], the assumption is even weaker: the system should be genuinely nonlinear, meaning that the diagonal coefficients of the matrix $\mathcal{A}$ are positive (a condition obviously weaker than assuming that $\mathcal{A}$ is definite positive, not to mention our coercivity assumption above.)

Not all Lax entropies are convex, or weak entropies - i.e. vanish for $\rho=0$. The reason why we have introduced this notion of admisssible solution of the isentropic Euler system in an open domain in space-time is the need for a control of the entropy production

$$
\partial_{t} \phi_{ \pm}(w, k)+\partial_{x} \psi_{ \pm}(w, k)=:-\mu_{ \pm}^{k}
$$

in terms of the energy dissipation.

Perhaps the same regularizing effect can be obtained by using only weak entropies - as in the original proof of compactness by DiPerna. This would require refining significantly the present argument, and is the subject of ongoing investigations.

Finally, it would be also interesting to know whether compensated compactness can be used to prove regularization estimates for hyperbolic problems - scalar conservation laws - in space dimension higher than one. Recent results, such as TRB, KRT suggest that this could be the case. 
Acknowledgements. I wish to express my gratitude to Yann Brenier, Camillo De Lellis, Peter Lax, Benoit Perthame and Eitan Tadmor for many illuminating discussions on the topics discussed in this paper.

\section{References}

[A] V. I. Agoshkov, Spaces of functions with differential-difference characteristics and the smoothness of solutions of the transport equation, Dokl. Akad. Nauk SSSR 276 (1984), no. 6, 12891293.

[BGP] F. Bouchut, F. Golse and M. Pulvirenti, Kinetic equations and asymptotic theory. Edited and with a foreword by Benoît Perthame and Laurent Desvillettes. Series in Applied Mathematics (Paris), 4. Gauthier-Villars, Editions Scientifiques et Médicales Elsevier, Paris, 2000.

[Chen] G.Q. Chen, Remarks on R. J. DiPerna's paper: "Convergence of the viscosity method for isentropic gas dynamics”, Proc. Amer. Math. Soc. 125 (1997), no. 10, 2981-2986.

[Chev] C. Cheverry, Regularizing effects for multidimensional scalar conservation laws, Ann. Inst. H. Poincaré Anal. Non Linéaire 17 (2000), no. 4, 413-472.

[Conw] E.D. Conway, The formation and decay of shocks for a conservation law in several dimensions, Arch. Rational Mech. Anal. 64 (1977), no. 1, 47-57.

[D1985] C. Dafermos, Regularity and large time behaviour of solutions of a conservation law without convexity, Proc. Roy. Soc. Edinburgh A 99 (1985), 201-239.

[D2000] C. Dafermos, Hyperbolic conservation laws in continuum physics, Grundlehren der Mathematischen Wissenschaften, 325. Springer-Verlag, Berlin, 2000.

[DL-G] C. DeLellis, F. Golse, A quantitative compactness estimate for scalar conservation laws, Comm. Pure Appl. Math. 58 (2005), no. 7, 989-998.

[DL-W] C. DeLellis, M. Westdickenberg, On the optimality of velocity averaging lemmas, Ann. Inst. H. Poincaré Anal. Non Linéaire 20 (2003), no. 6, 1075-1085.

[DP1983a] R.J. DiPerna, Convergence of the viscosity method for isentropic gas dynamics, Comm. Math. Phys. 91 (1983), no. 1, 1-30.

[DP1983b] R.J. DiPerna, Convergence of approximate solutions to conservation laws, Arch. Rational Mech. Anal. 82 (1983), no. 1, 27-70.

[DP1985] R.J. DiPerna, Compensated compactness and general systems of conservation laws, Trans. Amer. Math. Soc. 292 (1985), no. 2, 383-420.

[DP-L-M] R.J. DiPerna, P.-L. Lions, Y. Meyer, $L^{p}$ regularity of velocity averages, Ann. Inst. H. Poincaré Anal. Non Linéaire 8 (1991), no. 3-4, 271-287.

[GL] J. Glimm, P.D. Lax, Decay of solutions of systems of nonlinear hyperbolic conservation laws, Memoirs of the American Mathematical Society, No. 101 American Mathematical Society, Providence, R.I. 1970.

[Gér] P. Gérard, Microlocal defect measures, Comm. Partial Diff. Eq. 16 (1991), 1761-1794.

[Go] F. Golse, Compensated compactness and regularizing effects for hyperbolic conservation laws, preprint.

[GPS] F. Golse, B. Perthame, R. Sentis, Un résultat de compacité pour les équations de transport et application au calcul de la limite de la valeur propre principale d'un opérateur de transport, C. R. Acad. Sci. Paris Sér. I Math. 301 (1985), no. 7, 341-344.

[GLPS] F. Golse, P.-L. Lions, B. Perthame, R. Sentis, Regularity of the moments of the solution of a transport equation, J. Funct. Anal. 76 (1988), no. 1, 110-125.

[JP] P.-E. Jabin, B. Perthame, Regularity in kinetic formulations via averaging lemmas, A tribute to J. L. Lions, ESAIM Control Optim. Calc. Var. 8 (2002), 761-774.

[KRT] K. Karlsen, M. Rascle, E. Tadmor, On the existence and compactness of a two-dimensional resonant system of conservation laws, Commun. Math. Sci. 5 (2007), no. 2, 253-265.

[KT] A.N. Kolmogorov, V.M. Tikhomirov, $\varepsilon$-entropy and $\varepsilon$-capacity of sets in function spaces, Uspehi Mat. Nauk 14 (1959) no. 2 (86), 3-86.

[L1954] P.D. Lax, Weak solutions of nonlinear equations and their numerical computation, Comm. Pure and Appl. Math. 7 (1954), 159-194.

[L1957] P.D. Lax, Hyperbolic systems of conservation laws II, Comm. Pure and Appl. Math. 10 (1957), 537-566.

[L1971] P.D. Lax, Shock waves and entropy, Contributions to nonlinear functional analysis (Proc. Sympos., Math. Res. Center, Univ. Wisconsin, Madison, Wis., 1971), pp. 603-634. Academic Press, New York, 1971. 
[L1973] P.D. Lax, Hyperbolic systems of conservation laws and the mathematical theory of shock waves Conf. Board of the Math. Sciences, Regional Conf. Series in Appl. Math. (SIAM), 1973.

[L1978] P.D. Lax, Accuracy and resolution in the computation of solutions of linear and nonlinear equations, in Recent Adv. in Numer. Anal., Proc. of Symp., Madison (Academic Press), 107117.

[LPS] P.-L. Lions, B. Perthame, P. Souganidis, Existence and stability of entropy solutions for the hyperbolic systems of isentropic gas dynamics in Eulerian and Lagrangian coordinates, Comm. Pure Appl. Math. 49 (1996), no. 6, 599-638.

[LPT-a] P.-L. Lions, B. Perthame, E. Tadmor, A kinetic formulation of multidimensional scalar conservation laws and related equations, J. Amer. Math. Soc. 7 (1994), no. 1, 169-191.

[LPT-b] P.-L. Lions, B. Perthame, E. Tadmor, Kinetic formulation of the isentropic gas dynamics and p-systems, Comm. Math. Phys. 163 (1994), no. 2, 415-431.

[M] F. Murat, Compacité par compensation, Ann. Scuola Norm. Sup. Pisa Cl. Sci. (4) 5 (1978), no. 3, 489-507.

[O] O.A. Oleinik, On discontinuous solutions of nonlinear differential equations, Doklady Akad. Nauk SSSR, 109 (1956), 1098-1101.

[TRB] E. Tadmor, M. Rascle, P. Bagnerini, Compensated compactness for $2 D$ conservation laws, J. Hyperbolic Differ. Equ. 2 (2005), no. 3, 697-712.

[TT] E. Tadmor, T. Tao, Velocity averaging, kinetic formulations, and regularizing effects in quasi-linear PDEs, Comm. Pure Appl. Math. 60 (2007), no. 10, 1488-1521.

[T] L. Tartar, Compensated compactness and applications to partial differential equations, Nonlinear analysis and mechanics: Heriot-Watt Symposium, Vol. IV, pp. 136-212, Res. Notes in Math., 39, Pitman, Boston, Mass.-London, 1979.

Centre de mathématiques Laurent Schwartz, Ecole polytechnique, 91128 Palaiseau Cedex, France

E-mail address: francois.golse@math.polytechnique.fr 